\title{
Association between hyperuricemia and atrial fibrillation in rural China: a cross-sectional study
}

\author{
Guo-Zhe Sun', Liang Guo ${ }^{1}$, Jun Wang ${ }^{1}$, Ning Ye ${ }^{1}$, Xun-Zhang Wang ${ }^{2}$ and Ying-Xian Sun ${ }^{*}$
}

\begin{abstract}
Background: To explore the association between atrial fibrillation (AF) and serum uric acid (SUA) in a general population in rural China.

Methods: From January 2013 to August 2013, we performed a cross-sectional study involving 11,956 permanent residents $\geq 35$ years old in the rural Liaoning province of China. All participants completed a questionnaire, had a physical examination, and underwent an electrocardiogram (ECG) and echocardiogram. AF was diagnosed from ECG findings and/or a history of physician-confirmed AF. Blood samples were drawn for laboratory analyses and hyperuricemia was defined as an SUA level $>7.0 \mathrm{mg} / \mathrm{dL}$ in men and $>5.7 \mathrm{mg} / \mathrm{dL}$ in women, based on the NHANES-III laboratory definition. Logistic regression analyses were performed to estimate the crude and independent associations between hyperuricemia and the prevalence of AF.

Results: A total of 139 participants were diagnosed with AF, of which, 72 were self-reported, 45 were ECG-diagnosed, and 22 were both. There was a higher prevalence of $A F$ in participants with hyperuricemia than those with normal SUA levels (2.4 vs. $1.0 \% ; P<0.001)$. The odds ratios (OR) and $95 \%$ confidence intervals (Cl) were 2.37 (1.61-3.49) when compared to participants with normal SUA. After adjustment for other cardiovascular and AF risk factors, the independent association remained ( $\mathrm{OR}=1.94,95 \% \mathrm{Cl}$ : 1.26-3.00). Similar associations were observed between SUA as a continuous variable and AF prevalence (adjusted $\mathrm{OR}=1.20,95 \% \mathrm{Cl}: 1.06-1.36$ ). The independent associations were significant in men $\left(P_{S}<0.05\right)$ but not in women $\left(P_{s}>0.05\right)$, although the interaction logistic regression analyses presented these differences as not being statistically significant ( $\left.P_{S}>0.05\right)$.
\end{abstract}

Conclusions: SUA is positively associated with the prevalence of AF in rural China.

\section{Background}

Atrial fibrillation (AF) is one of the most common cardiac arrhythmias and is associated with overall mortality and mortality from cardiovascular disease [1, 2]. Advancing age, male gender, hypertension, diabetes mellitus, obesity, heart failure, myocardial infarction (MI), and alcohol consumption are the major risk factors for the development of AF [3-6]. The prevalence of AF is expected to increase dramatically over the next few decades as the general population ages and improved cardiovascular therapies keep people with cardiovascular disease alive longer [7]. Identifying all the risk factors for AF will help to create population-based strategies to deal with this serious health problem.

\footnotetext{
* Correspondence: cmu1h_syx@126.com

${ }^{1}$ Department of Cardiovascular Medicine, The First Hospital of China Medical University, 155 Nanjing Street, Heping, Shenyang, Liaoning 110001, China Full list of author information is available at the end of the article
}

Serum uric acid (SUA) is a risk factor for cerebrovascular and coronary artery disease, as well as for hypertension, metabolic syndrome, and kidney disease [8], though only a few cross-sectional studies in Japan [9], Turkey [10], and China [11-13] have reported a positive association between hyperuricemia and the prevalence of AF. Also, population-based prospective cohorts showed that hyperuricemia was associated with a high risk of AF $[14,15]$. However, all of these cross-sectional studies enrolled hospital patients only rather than individuals from the general population. Furthermore, only the study in Japan analyzed the effect of gender on the association between hyperuricemia and AF, reporting that the independent association was observed in women only. Therefore, the current study was designed to explore the association between SUA and AF in a general population from rural China. The study also analyzed the effect of gender on the independent association. 


\section{Methods}

\section{Study population}

A representative sample of men and women $\geq 35$ years of age from rural areas of Liaoning Province were recruited between January 2013 and August 2013 using a multistage, randomly stratified, cluster-sampling scheme. In particular, three counties (Dawa, Zhangwu, and Liaoyang) were randomly selected from Liaoning Province. One township near a city in each county was randomly selected giving a total of three townships. Six to eight villages from each township were randomly selected to give a total of 26 rural villages. All of the eligible permanent residents aged $\geq 35$ years from each village $(n=14,016)$ were invited to participate in the study, and $11,956(85.3 \%)$ agreed to do so; women who were pregnant, or people who had cancer or any mental disorders were excluded from the study.

The study was carried out with pre-approval granted by the Ethics Committee of China Medical University (Shenyang, China). Written consent was obtained from all participants after they had been informed of the study's objectives, benefits, medical procedures, and confidentiality safeguards for personal information. In the case of an illiterate participant, written informed consent was obtained from the appropriate legal proxy.

\section{Data collection and measurement}

Data were collected during a single clinic visit by cardiologists and trained nurses using a standard questionnaire in a face-to-face interview. All potential investigators had received training on the objectives of the study, how to administer the questionnaire, the standard methods of measurement, the importance of standardization, and study procedures. Only those who earned a perfect score on a post-training test were allowed to participate as study investigators. During data collection, the inspectors received further instructions and support.

Data on demographic characteristics and medical history of AF, MI, hypertension, diabetes mellitus, lifestyle risk factors, and family history of AF were obtained, as described above, by interview with the standardized questionnaire. There was a central steering committee with a subcommittee for quality control that made sure all data were collected according to well-known standards.

According to the American Heart Association, blood pressure (BP) was measured three times at two-minute intervals after at least five minutes of rest using a standardized automatic electronic sphygmomanometer (HEM-907; Omron, Kyoto, Japan). Two doctors checked the calibration of the Omron device every month using a standard mercury sphygmomanometer according to the British Hypertension Society protocol [16]. The participants were advised to avoid caffeinated beverages and to exercise for $\geq 30 \mathrm{~min}$ before the measurement. During the measurement, the participants were seated with their arms supported at the level of their hearts. The mean of three BP measurements was calculated and used in all analyses.

Weight and height were measured to the nearest $0.1 \mathrm{~kg}$ and $0.1 \mathrm{~cm}$, respectively, with the participants in lightweight clothing without shoes. The body mass index (BMI) was calculated as weight in kilograms divided by the square of the height in meters. Waist circumference (WC) was measured at the umbilicus to the nearest $0.1 \mathrm{~cm}$ while the participants were standing following a normal expiration.

Fasting blood samples were collected in the morning after $\geq 8 \mathrm{~h}$ of fasting for all participants. Blood samples were obtained from an antecubital vein using BD Vacutainer tubes containing EDTA (Becton, Dickinson and Co., Franklin Lakes, NJ, USA). Serum was subsequently isolated from whole blood, and all serum samples were frozen at $-20{ }^{\circ} \mathrm{C}$ for testing at a central, certified laboratory. Fasting blood glucose (FBG), total cholesterol (TC), triglycerides (TG), SUA, and other routine blood biochemical indices were analyzed enzymatically on an autoAnalyzer (Olympus AU640; Olympus, Kobe, Japan, or Bayer RA-XT; Bayer Diagnostics, Tarrytown, NY, USA) using kits (Bayer Diagnostics). The laboratory measurements were calibrated and verified following analysis of biochemical indices and the results met the national standards of measurement (CNAS certificate of accreditation No.L0467, quality index $U=0.006(\mathrm{k}=2)$ ).

Twelve-lead resting, ten-second electrocardiograms (ECGs) were performed on all participants by well-trained cardiologists using an electrocardiography machine (MAC 5500; GE Healthcare, Little Chalfont, Buckinghamshire, UK). The results were analyzed automatically by the MUSE Cardiology Information System (version 7.0.0; GE Healthcare). ECG-based diagnoses of AF were confirmed by at least two independent cardiologists.

Echocardiograms were obtained using a commercially available Doppler echocardiograph (Vivid; GE Healthcare) with a $3.0-\mathrm{MHz}$ transducer. The transthoracic echocardiogram included M-mode, two-dimensional, spectral and color Doppler with subjects in the supine position. Echocardiogram analyses and readings were performed by three doctors specialized in echocardiography, and two other specialists were called in if questions or uncertainty arose. Measurements were performed according to the recommendations of the American Society of Echocardiography. M-mode images were used to measure and calculate the left ventricular ejection fraction (LVEF) [17].

\section{Definitions}

AF was diagnosed based on a previous history of AF (previously diagnosed by a physician) and/or evidence of AF on the ECG (absence of consistent P waves, presence of rapid irregular $f$ waves with a frequency of 350-600 beats per minute, and an irregular ventricular response). 
Left ventricular hypertrophy diagnosed by ECG (ECGLVH) was identified using the Cornell Criteria expressed as voltage and QRS duration product: $(\mathrm{RaVL}+\mathrm{SV} 3) \times$ QRS duration $>2,440 \mathrm{~mm} * \mathrm{~ms}$ in men and $(\mathrm{RaVL}+\mathrm{SV} 3+$ $8 \mathrm{~mm}) \times$ QRS duration $>2,440 \mathrm{~mm}^{*} \mathrm{~ms}$ in women [18]. Left ventricular systolic dysfunction was defined as an LVEF $<0.5$ based on M-mode echocardiography. Hyperuricemia was defined as an SUA level $>7.0 \mathrm{mg} / \mathrm{dL}$ in men and $>5.7 \mathrm{mg} / \mathrm{dL}$ in women, based on the NHANES-III laboratory definitions [19].

\section{Statistical analysis}

All statistical analyses were performed using SPSS 17.0 software (SPSS Inc., Chicago, IL, USA). Differences between groups were compared using a two-tailed Student's $t$-test for continuous variables and a $x^{2}$ test for categorical variables. The age- and gender-specific prevalences of AF among participants with both normal SUA levels and hyperuricemia were calculated, and univariate and multivariate logistic regression analyses were performed to estimate the crude and independent association between SUA and the presence of AF. Interaction regression models were used to test the difference in the association of SUA with AF prevalence between men and women. Data are expressed as odds ratio (OR) and $95 \%$ confidence interval $(\mathrm{CI})$, mean \pm standard deviation, or frequency and percentage; a $P<0.05$ was considered as statistically significant.

\section{Results}

\section{Characteristics of the study population}

Of the original 11,956 participants, 618 had incomplete data and were excluded from the analysis, leaving a total of 11,338 participants (5,170 men and 6,168 women) with a mean age of 53.8 years. The subjects with hyperuricemia were older than those with normal SUA levels $(P<0.001)$, and there was a higher percentage of men than women in this group $(P<0.001)$ (Table 1$)$. Participants with hyperuricemia had significantly higher WCs, BMIs, systolic and diastolic BPs, FBG, TC, and TG levels (all $P_{\mathrm{S}}<0.001$ ). The hyperuricemia group also had a higher percentage of alcohol drinkers and had a higher prevalence of MI, and ECG-LVH than the normal SUA level group (all $P_{\mathrm{s}}<0.001$ ). The prevalence of AF was significantly higher in participants with hyperuricemia than those with normal SUA $(P<0.001)$.

\section{Prevalence of AF by SUA level}

There were 139 participants with $\mathrm{AF}$, and the agespecific prevalence of AF by SUA level is summarized in Fig. 1. Among these AF cases, 72 were self-reported, 45 were ECG-diagnosed, and 22 were both. The prevalence of AF rose steeply with advancing age and was higher in the group with hyperuricemia than in the group with
Table 1 Characteristics of the study population

\begin{tabular}{lllr}
\hline Variable & $\begin{array}{l}\text { Normal SUA level } \\
(n=9,909)\end{array}$ & $\begin{array}{l}\text { Hyperuricemia } \\
(n=1,429)\end{array}$ & $P$ \\
\hline Age, y & $53.7 \pm 10.5$ & $54.9 \pm 10.9$ & $<0.001$ \\
Sex, male & $4396(44.4)$ & $774(54.2)$ & $<0.001$ \\
BMI, kg/m & $24.6 \pm 3.6$ & $26.4 \pm 3.9$ & $<0.001$ \\
WC, cm & $81.6 \pm 9.6$ & $87.9 \pm 9.9$ & $<0.001$ \\
SBP, mmHg & $141.2 \pm 23.4$ & $145.9 \pm 23.8$ & $<0.001$ \\
DBP, mmHg & $81.5 \pm 11.5$ & $85.7 \pm 12.7$ & $<0.001$ \\
FBG, mmol/L & $5.88 \pm 1.65$ & $6.05 \pm 1.47$ & $<0.001$ \\
TC, mmol/L & $5.18 \pm 1.06$ & $5.58 \pm 1.23$ & $<0.001$ \\
TG, mmol/L & $1.53 \pm 1.30$ & $2.41 \pm 2.34$ & $<0.001$ \\
Current smoker & $3478(35.1)$ & $502(35.1)$ & 0.982 \\
CUrrent drinker & $2107(21.3)$ & $418(29.3)$ & $<0.001$ \\
History of Ml & $101(1.0)$ & $30(2.1)$ & $<0.001$ \\
LVEF $<0.5$ & $1126(11.8)$ & $144(10.4)$ & 0.131 \\
ECG-LVH & $799(8.1)$ & $215(15.0)$ & $<0.001$ \\
AF & $104(1.0)$ & $35(2.4)$ & $<0.001$ \\
Family history of AF & $306(3.1)$ & $38(2.7)$ & 0.377 \\
\hline Nete data & & &
\end{tabular}

Note: data are expressed as mean \pm standard deviation or $n(\%)$ $A F$ atrial fibrillation, $B M I$ body mass index, $D B P$ diastolic blood pressure, $E C G-L V H$ left ventricular hypertrophy detected by electrocardiography, $F B G$ fasting blood glucose, $L V E F$ left ventricular ejection fraction, $M I$ myocardial infarction, SBP systolic blood pressure, SUA serum uric acid, TC total cholesterol, $T G$ triglycerides, $W C$ waist circumference

normal SUA levels at every age. The trends in AF prevalence with age were similar between men and women.

\section{Association between SUA and AF}

The association between hyperuricemia and AF was examined by logistic regression analysis (Table 2). Both men and women with hyperuricemia had a higher prevalence of AF than those with normal SUA levels $(P<$ 0.001 for men, $P=0.010$ for women and $P<0.001$ for total). After adjusting for other cardiovascular and AF risk factors, including age, gender, WC, BMI, systolic and diastolic BP, FBG, TC, TG, smoking, drinking, history of MI, low LVEF, ECG-LVH and familial history of AF, the independent association was significant in men $(P=0.003)$ and in men and women together $(P=0.003)$, but not in women alone $(P=0.235)$. However, $P>0.05$ was observed for the association of AF with hyperuricemia between men and women. Similar associations were found between SUA as a continuous variable and AF prevalence (Table 3).

\section{Discussion}

The results of this study demonstrate that men and women with hyperuricemia in a rural Chinese population have a significantly higher prevalence of AF than those with normal SUA levels. The AF prevalence increases with advancing age in both men and women, 


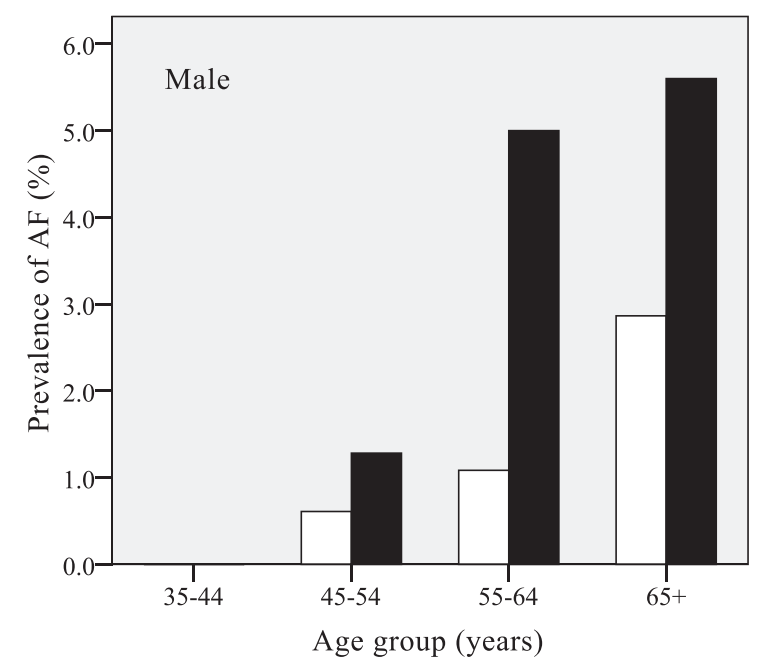

$\square$ Normouricemia

Hyperuricemia

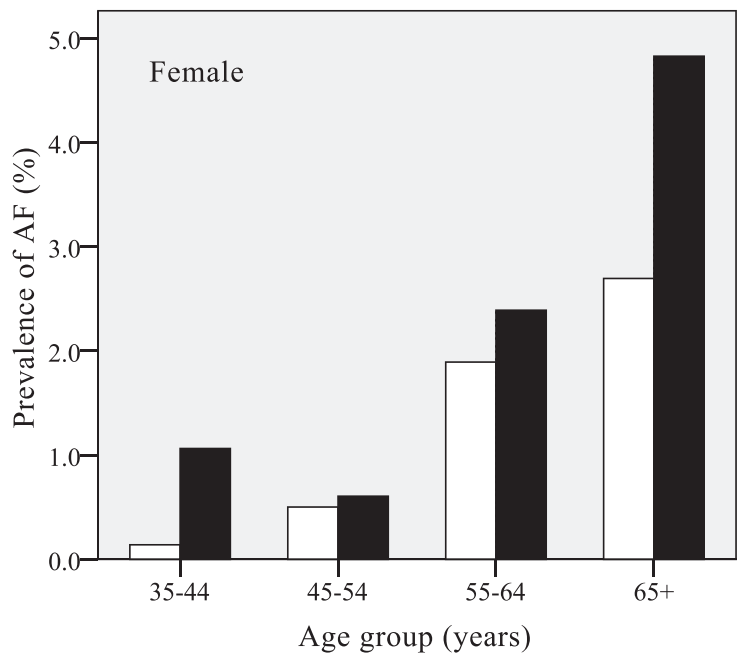

Normouricemia

Hyperuricemia

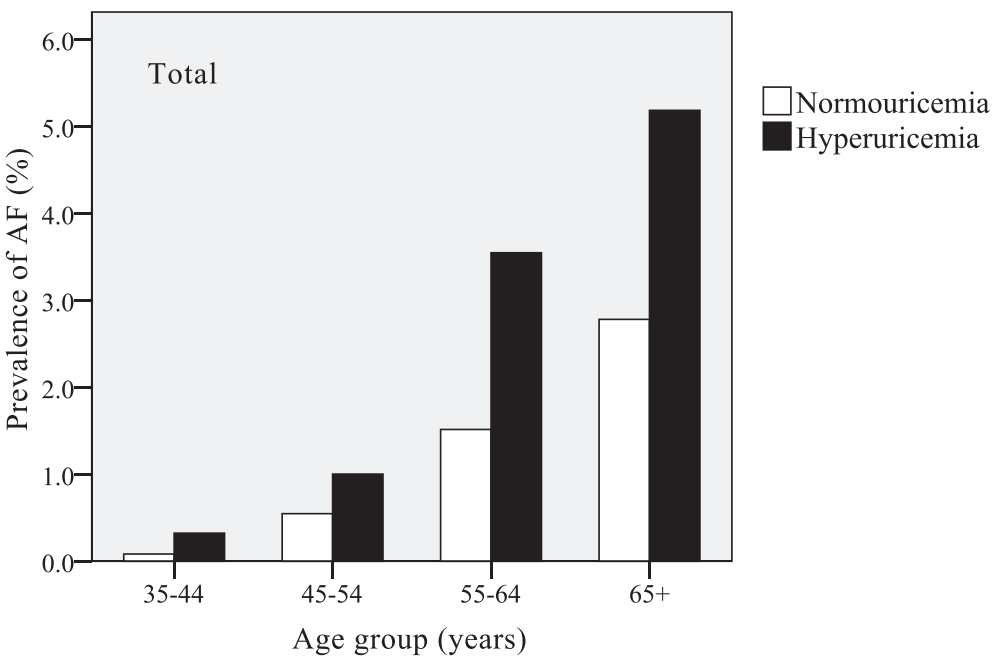

Fig. 1 Age-specific prevalence of atrial fibrillation by serum uric acid levels. The prevalence of AF in men (top), women (center), and the total population (bottom) 
Table 2 Hyperuricemia and the prevalence of atrial fibrillation

\begin{tabular}{|c|c|c|c|c|c|c|}
\hline \multirow[t]{2}{*}{ Group } & \multirow{2}{*}{$\begin{array}{l}\text { Total } \\
(n)\end{array}$} & \multirow{2}{*}{$\begin{array}{l}\text { AF } \\
n(\%)\end{array}$} & \multicolumn{2}{|c|}{ Unadjusted model } & \multicolumn{2}{|l|}{ Adjusted model $^{a}$} \\
\hline & & & OR $(95 \%$ Cl) & $P$ & OR $(95 \%$ Cl) & $P$ \\
\hline \multicolumn{7}{|l|}{ Men } \\
\hline Normal SUA levels & 4,396 & $44(1.0)$ & 1 & $<0.001$ & 1 & 0.003 \\
\hline Hyperuricemia & 774 & $20(2.6)$ & $2.62(1.54-4.48)$ & & $2.55(1.38-4.71)$ & \\
\hline \multicolumn{7}{|l|}{ Women } \\
\hline Normal SUA levels & 5,513 & $60(1.1)$ & 1 & 0.010 & 1 & $0.235^{*}$ \\
\hline Hyperuricemia & 655 & $15(2.3)$ & $2.13(1.20-3.77)$ & & $1.46(0.78-2.72)$ & \\
\hline \multicolumn{7}{|l|}{ Total } \\
\hline Normal SUA levels & 9,909 & $104(1.0)$ & 1 & $<0.001$ & 1 & 0.003 \\
\hline Hyperuricemia & 1,429 & $35(2.4)$ & $2.37(1.61-3.49)$ & & $1.94(1.26-3.00)$ & \\
\hline
\end{tabular}

$A F$ atrial fibrillation, $\mathrm{Cl}$ confidence interval, $O R$ odds ratio, SUA serum uric acid

${ }^{*} P=0.200$ for gender difference

${ }^{a}$ Adjusted for age, gender, body mass index, waist circumference, systolic and diastolic blood pressure, fasting blood glucose, total cholesterol and triglyceride levels, smoking, drinking, myocardial infarction, low left ventricular ejection fraction, left ventricular hypertrophy detected by electrocardiography, and family history of AF

but the independent association with SUA is only observed in the total population and in men after adjusting for other cardiovascular risk factors. Our finding that hyperuricemia was positively associated with the AF prevalence in the general population was consistent with previous studies [9-13]. However, in our current study, the independent association was only observed in men but not in women, which was inconsistent with previous findings in Japan [9]. A recent meta-analysis that included both cross-sectional and cohort studies, also reported a positive relationship between SUA and the prevalence of AF [20]. Population-based prospective cohort studies showed that baseline SUA was associated with an increased risk of AF in both genders [14, 15]. It is possible that race and lifestyle influenced the results

Table 3 Serum uric acid levels and the prevalence of atrial fibrillation

\begin{tabular}{|c|c|c|c|c|}
\hline \multirow[t]{2}{*}{ Group } & \multicolumn{2}{|c|}{ Unadjusted model } & \multicolumn{2}{|l|}{ Adjusted model $^{a}$} \\
\hline & OR $(95 \%$ Cl) & $P$ & OR $(95 \% \mathrm{Cl})$ & $P$ \\
\hline \multicolumn{5}{|l|}{ Men } \\
\hline SUA, per mg/dl & $1.30(1.13-1.50)$ & $<0.001$ & $1.26(1.08-1.47)$ & 0.004 \\
\hline \multicolumn{5}{|l|}{ Women } \\
\hline SUA, per mg/dl & $1.31(1.11-1.55)$ & 0.002 & $1.11(0.92-1.33)$ & $0.291^{*}$ \\
\hline \multicolumn{5}{|l|}{ Total } \\
\hline SUA, per mg/dl & $1.25(1.13-1.38)$ & $<0.001$ & $1.20(1.06-1.36)$ & 0.003 \\
\hline
\end{tabular}

$A F$ atrial fibrillation, $\mathrm{Cl}$ confidence interval, $O R$ odds ratio, SUA serum uric acid ${ }^{*} P=0.302$ for gender difference

${ }^{a}$ Adjusted for age, gender, body mass index, waist circumference, systolic and diastolic blood pressure, fasting blood glucose, total cholesterol and

triglyceride levels, smoking, drinking, myocardial infarction, low left ventricular ejection fraction, left ventricular hypertrophy detected by electrocardiography, and family history of AF of our study, as Asians and African-Americans have a lower prevalence of AF than other races [21], and our study population consisted of people living exclusively in rural areas, most of whom routinely performed heavy physical labor.

We also tested the difference in the association of SUA with AF between men and women by interaction regression models. However, the effect of gender on the association was not statistically significant with $P s>0.05$. This inconsistency may be due to low AF prevalence and the small sample size. Indeed, the positive association between SUA and AF might also exist in women (even though this might not be statistically significant).

In our current study, AF was diagnosed based on a previous history of physician-diagnosed AF and/or evidence of AF using the ECG. This diagnostic approach has been utilized in previous studies [9, 22]. Selfreported AF history was previously used as a method for the diagnosis of AF. However, it is likely that this approach gave rise to an unintentional bias.

The major clinical risk factors for AF are advancing age, male gender, hypertension, diabetes, obesity, heart failure, valve disease, MI, smoking, and alcohol consumption, but biomarkers such as B-type natriuretic peptide $[23,24]$ and C-reactive protein $[25,26]$ also correlate with AF. Systemic inflammation indicated by an elevated level of $\mathrm{C}$-reactive protein is associated with the incidence and persistence of AF [26, 27]. SUA regulates some critical proinflammatory pathways [28] and correlates with several inflammatory markers [29]. Inflammation could cause oxidative damage to the atrium that might contribute to electrical remodeling and increase the incidence of AF [30]. SUA may therefore be another useful biomarker for the condition, though 
the mechanisms by which SUA influences AF risk are unclear.

A limitation of this study is the cross-sectional design and it is not clear whether lowering SUA levels would also decrease the incidence and prevalence of AF. In addition, the number of people who had hyperuricemia in our study was small, and the prevalence of $\mathrm{AF}$ in some subgroups was zero or too small to measure for statistical significance. The association between SUA and AF in our study may also have been affected by confounding risk factors for AF (i.e., hypertension, diabetes, and dislipidemia), which also occur with hyperuricemia. However, results from the univariate and multivariate logistic regression analyses indicate that SUA is an independent risk factor for AF. But, further cohort studies need to be conducted to determine whether reducing SUA levels reduce AF incidence and prevalence.

\section{Conclusions}

Men and women in rural China $\geq 35$ years of age with hyperuricemia have a significantly higher prevalence of AF than those with normal SUA levels. Hyperuricemia is independently associated with the prevalence of AF after adjusting for various cardiovascular risk factors.

\section{Competing interests}

The authors declare that they have no competing interests.

\section{Authors' contributions}

GZS collected the data, analyzed and prepared the first draft of the manuscript. LG supervised the data collection and reviewed the manuscript. JW collected the data. NY coordinated the data collection. XZW reviewed the analysis and the manuscript. YXS conceived the study design, reviewed the manuscript and serves as guarantor for the contents of this paper. All authors approved the final version.

\section{Acknowledgments}

This study was founded by the National Science and Technology Support Program of China (No. 2012BAJ18B08-7).

\section{Grant support}

National Science and Technology Support Program of China (No. 2012BAJ18B08-7).

\section{Author details}

'Department of Cardiovascular Medicine, The First Hospital of China Medical University, 155 Nanjing Street, Heping, Shenyang, Liaoning 110001, China. ${ }^{2}$ Heart Institute, Cedars Sinai Medical Center, Los Angeles 90048, CA, USA.

Received: 10 December 2014 Accepted: 20 August 2015

Published online: 01 September 2015

\section{References}

1. Kannel WB, Abbott RD, Savage DD, McNamara PM. Epidemiologic features of chronic atrial fibrillation: the Framingham study. N Engl J Med. 1982;306:1018-22.

2. Stewart S, Hart CL, Hole DJ, McMurray JJ. A population-based study of the long-term risks associated with atrial fibrillation: 20-year follow-up of the Renfrew/Paisley study. Am J Med. 2002;113:359-64.

3. Benjamin EJ, Levy D, Vaziri SM, D'Agostino RB, Belanger AJ, Wolf PA. Independent risk factors for atrial fibrillation in a population-based cohort. The Framingham Heart Study. JAMA. 1994;271:840-4.
4. Korantzopoulos P, Kolettis TM. Obesity and the risk of new-onset atrial fibrillation. JAMA. 2005;293:1974-5.

5. Schnabel RB, Sullivan LM, Levy D, Pencina MJ, Massaro JM, D'Agostino Sr RB, et al. Development of a risk score for atrial fibrillation (Framingham Heart Study): a community-based cohort study. Lancet. 2009;373:739-45.

6. Djoussé L, Levy D, Benjamin E, Blease SJ, Russ A, Larson MG, et al. Long-term alcohol consumption and the risk of atrial fibrillation in the Framingham Study. Am J Cardiol. 2004;93:710-3.

7. Miyasaka Y, Barnes ME, Gersh BJ, Cha SS, Bailey KR, Abhayaratna WP, et al. Secular trends in incidence of atrial fibrillation in Olmsted County, Minnesota, 1980 to 2000, and implications on the projections for future prevalence. Circulation. 2006;114:119-25.

8. Feig DI, Kang DH, Johnson RJ. Uric acid and cardiovascular risk. N Engl J Med. 2008:359:1811-21.

9. Suzuki S, Sagara K, Otsuka T, Matsuno S, Funada R, Uejima T, et al. Gender-specific relationship between serum uric acid level and atrial fibrillation prevalence. Circ J. 2012;76:607-11.

10. Tekin G, Tekin YK, Erbay AR, Turhan H, Yetkin E. Serum uric acid levels are associated with atrial fibrillation in patients with ischemic heart failure. Angiology. 2013;64:300-3.

11. Liu Y, Liu H, Dong L, Chen J, Guo J. Prevalence of atrial fibrillation in hospitalized patients over 40 years old: ten-year data from the People's Hospital of Peking University. Acta Cardiol. 2010;65:221-4.

12. Zhao QY, Yu SB, Huang H, Cui HY, Qin M, Huang T, et al. Serum uric acid levels correlate with atrial fibrillation in patients with chronic systolic heart failure. Chin Med J (Engl). 2012;125:1708-12.

13. Liu T, Zhang X, Korantzopoulos P, Wang S, Li G. Uric acid levels and atrial fibrillation in hypertensive patients. Intern Med. 2011;50:799-803.

14. Nyrnes A, Toft I, Njølstad I, Mathiesen EB, Wilsgaard T, Hansen JB, et al. Uric acid is associated with future atrial fibrillation: an 11-year follow-up of 6308 men and women-the Tromso Study. Europace. 2014;16:320-6.

15. Tamariz L, Agarwal S, Soliman EZ, Chamberlain AM, Prineas R, Folsom AR, et al. Association of serum uric acid with incident atrial fibrillation (from the Atherosclerosis Risk in Communities [ARIC] study). Am J Cardiol. 2011;108:1272-6.

16. O'Brien E, Petrie J, Littler W, de Swiet M, Padfield PL, O'Malley K, et al. The British Hypertension Society protocol for the evaluation of automated and semi-automated blood pressure measuring devices with special reference to ambulatory systems. J Hypertens. 1990;8:607-19.

17. Sahn DJ, DeMaria A, Kisslo J, Weyman A. Recommendations regarding quantitation in M-mode echocardiography: results of a survey of echocardiographic measurements. Circulation. 1978;58:1072-83.

18. Dahlöf B, Devereux RB, Julius S, Kjeldsen SE, Beevers G, de Faire U, et al. Characteristics of 9194 patients with left ventricular hypertrophy: the LIFE study. Losartan Intervention For Endpoint Reduction in Hypertension. Hypertension. 1998:32:989-97

19. Zhu Y, Pandya BJ, Choi HK. Prevalence of gout and hyperuricemia in the US general population: the National Health and Nutrition Examination Survey 2007-2008. Arthritis Rheum. 2011;63:3136-41.

20. Tamariz L, Hernandez F, Bush A, Palacio A, Hare JM. Association between serum uric acid and atrial fibrillation: a systematic review and meta-analysis. Heart Rhythm. 2014;11:1102-8.

21. Marcus GM, Olgin JE, Whooley M, Vittinghoff E, Stone KL, Mehra R, et al. Racial differences in atrial fibrillation prevalence and left atrial size. Am J Med. 2010;123:375.e1-7

22. Schnabel RB, Wilde S, Wild PS, Munzel T, Blankenberg S. Atrial fibrillation: its prevalence and risk factor profile in the German general population. Dtsch Arztebl Int. 2012;109:293-9.

23. Patton KK, Ellinor PT, Heckbert SR, Christenson RH, DeFilippi C, Gottdiener JS, et al. $\mathrm{N}$-terminal pro-B-type natriuretic peptide is a major predictor of the development of atrial fibrillation: the Cardiovascular Health Study. Circulation. 2009;120:1768-74.

24. Wang TJ, Larson MG, Levy D, Benjamin EJ, Leip EP, Omland T, et al. Plasma natriuretic peptide levels and the risk of cardiovascular events and death. N Engl J Med. 2004;350:655-63.

25. Cao JJ, Thach C, Manolio TA, Psaty BM, Kuller LH, Chaves PH, et al. C-reactive protein, carotid intima-media thickness, and incidence of ischemic stroke in the elderly: the Cardiovascular Health Study. Circulation. 2003;108:166-70.

26. Aviles RJ, Martin DO, Apperson-Hansen C, Houghtaling PL, Rautaharju P, Kronmal RA, et al. Inflammation as a risk factor for atrial fibrillation. Circulation. 2003;108:3006-10 
27. Issac T, Dokainish H, Lakkis NM. Role of inflammation in initiation and perpetuation of atrial fibrillation: a systematic review of the published data. J Am Coll Cardiol. 2007;50:2021-8.

28. Kanellis J, Watanabe S, Li JH, Kang DH, Li P, Nakagawa T, et al.

Uric acid stimulates monocyte chemoattractant protein-1 production in vascular smooth muscle cells via mitogen-activated protein kinase and cyclooxygenase-2. Hypertension. 2003;41:1287-93.

29. Ruggiero C, Cherubini A, Ble A, Bos AJ, Maggio M, Dixit VD, et al, Uric acid and inflammatory markers. Eur Heart J. 2006;27:1174-81.

30. Carnes CA, Chung MK, Nakayama T, Nakayama H, Baliga RS, Piao S, et al. Ascorbate attenuates atrial pacing-induced peroxynitrite formation and electrical remodeling and decreases the incidence of postoperative atrial fibrillation. Circ Res. 2001:89:E32-8.

\section{Submit your next manuscript to BioMed Central} and take full advantage of:

- Convenient online submission

- Thorough peer review

- No space constraints or color figure charges

- Immediate publication on acceptance

- Inclusion in PubMed, CAS, Scopus and Google Scholar

- Research which is freely available for redistribution 